\title{
Vacuum Electronics
}

\author{
Robert K. Parker, Fellow, IEEE, Richard H. Abrams, Jr., Member, IEEE, Bruce G. Danly, Senior Member, IEEE, \\ and Baruch Levush, Fellow, IEEE
}

Invited Paper

\begin{abstract}
This paper explores the recent history and diversity of this remarkable technology, with emphasis on recent advances in the more traditional device types (traveling-wave tube and klystron), as well as more recent innovations such as the microwave power module, inductive output amplifier, fast-wave devices, ultrahigh-power sources, and RF vacuum microelectronics. These advances can be credited to a combination of device innovation, enhanced understanding gained through improved modeling and design, the introduction of superior materials and sub-assembly components and the development of advanced vacuum processing and manufacturing techniques.
\end{abstract}

Index Terms-Cathodes, free-electron laser, gyrotron, high-power microwaves, klystron, microwave power module, numerical simulation, traveling-wave tube, TWT, vacuum electronics, vacuum microelectronics.

\section{INTRODUCTION}

$\mathbf{R}$ ADIO-FREQUENCY (RF) vacuum electronics continues to evolve at a revolutionary pace, a testament to its vibrancy and continuing relevance. For the past six decades, its capability to generate and amplify high-power coherent radiation at high frequency, as measured in terms of average output power density $\left(P_{\mathrm{av}} f^{2}\right)$, has continued to advance with a doubling time of two years. Modern RF vacuum amplifiers and oscillators find their primary role as sources and in transmitters operating in a spectral range extending from the UHF to the X-ray and providing peak powers spanning a range from tens of watts to tens of gigawatts. Through continued innovation and growth, RF vacuum electronics remains the technology of choice in a wide range of high-power microwave and millimeter-wave applications and offers a viable pathway for extending the full range of performance attributes with increased reliability and reduced cost.

Vacuum electronic amplifiers and oscillators are used in a wide variety of military and commercial applications requiring high power at high frequency, as well as in scientific research areas such as high-energy particle accelerators and plasma heating for controlled thermonuclear fusion where only vacuum electronic devices meet the requirements. In addition, vacuum amplifiers are widely used in many medical systems, such as compact RF accelerators and hypothermia applicators. Commercial satellite communication systems, broadcasting, and microwave ovens for industrial and home use

Manuscript received October 31, 2001.

The authors are with the Naval Research Laboratory, Washington, DC 20375-0001 USA.

Publisher Item Identifier S 0018-9480(02)01965-8. also rely heavily on vacuum devices for reliable performance at high power and high efficiency. The military has a critical reliance on vacuum amplifiers for radar, electronic warfare, and communications systems requiring high power, high efficiency, or high frequency. Currently, there are close to 185000 vacuum electronic device sockets, in over 270 U.S. military systems, and the number is still growing. Military interests also continue for the use of high-power microwave and millimeter-wave sources for directed energy applications.

Although the science of vacuum electronics has its origin in the electron beam physics of the early 20th Century, the RF vacuum electronics of today is far removed from the familiar image of the glass-envelope tube associated with early radio, television, and computer developments. More accurately, the technology of modern RF vacuum electronics is most closely akin to that of the high-energy particle accelerator. By employing robust metal-ceramic vacuum envelopes, lightweight rare-earth magnets and moderate-temperature dispenser cathodes, modern vacuum electronic amplifiers, and oscillators provide reliable and long-life operation.

The key themes defining modern RF vacuum electronics have their origin in the four-decade interval beginning in the mid1930s. The most familiar of the microwave power tube types were invented in the decade spanning the mid-1930s and 1940s. However, the birth of modern RF vacuum technology is more properly associated with several discoveries and technological advances made in the mid-1940s and early 1950s, contemporaneous with the invention of the transistor. Among these were the following:

1) the competitive emergence of the linear beam format;

2) innovation of periodic permanent magnet (PPM) focusing;

3) development of the depressed collector;

4) invention of the dispenser cathode;

5) implementation of simple metal/ceramic packaging.

From the richness of device concepts investigated through the 1960s, the helix and coupled cavity traveling wave tubes (TWTs), klystron, magnetron, and crossed field amplifier (CFA) emerged as the primary products of today's industry. This array of device types was augmented in the mid-1960s with the invention of the two principal classes of fast-wave device, i.e., the gyro-oscillator/amplifier and the free-electron laser (FEL). Ongoing development of these fast-wave devices has responded to the expanding need for power at millimeter and shorter wavelengths. The scope of the field was further 
expanded in the late 1960s by two themes emerging at the opposite extremes of power and physical size. Intrigued by the unique properties associated with relativistic and self-field effects, researchers began to study the use of intense relativistic electron beams (IREB) for the generation of ultrahigh-power pulses of microwave and millimeter-wave radiation. These intense electron beams became available with the development of pulsed high-current relativistic electron accelerators (10 MV $>V_{b}>0.1 \mathrm{MV}, P_{p k} \geq 1 \mathrm{GW}$, and $\tau \leq 0.1 \mu \mathrm{s}$ ) for the laboratory simulation of nuclear weapon effects. Finally, early work with field emission arrays (FEAs), during the same period, laid the foundation for a major sub-field described more recently as RF vacuum microelectronics.

In recent decades, vacuum electronics research has flourished with widespread efforts in the development of gyrotrons (gyro-oscillators) and FELs, the pursuit of ultrahigh-power device concepts and the exploration of hybrid concepts invoking microelectronic and microfabrication techniques. At the same time, the need for more power, better efficiency, higher frequency operation and wider bandwidth in a compact package pushed the field to new levels of performance through innovations in established amplifier types. These efforts were supported by advances in other technologies, such as computational hardware that provided the foundation for the development of physics-based design codes for vacuum devices and solid-state monolithic microwave integrated circuit (MMIC) amplifiers, which have provided the wide-band driver stage amplifier for the hybrid microwave power module (MPM) amplifier. Today, advances in power supply technology may provide the next step in noise and size reduction and refinement in materials, magnetics, and electron sources will contribute to improved performance, reduced cost, and increased reliability.

\section{Device Physics-The Basics}

The collision-free character of an electron beam propagating in vacuum focuses the challenge of device innovation on the physics of distributed beam-wave interaction mechanisms coupled with the details of circuit design, beam formation, and beam collection. The absence of collisions also allows the recovery of unused electron kinetic energy through the use of a depressed collector, thereby providing the vacuum device with a competitive advantage in terms of unsurpassed efficiency with bandwidth. Additionally, the presence of a depressed collector can be exploited to partially decouple constraints on efficiency and amplifier linearity.

Basically, vacuum electronic amplifiers and oscillators operate by converting spontaneous electromagnetic radiation into coherent radiation through a beam-wave interaction. The three basic mechanisms for electromagnetic radiation by charged particles [1] are as follows.

1) Cerenkov radiation occurs when electrons with uniform velocity pass through a medium (circuit or structure) in which components of the electromagnetic wave propagate with a phase velocity slower than the speed of the electrons (i.e., slower than the speed of light in a vacuum). Cerenkov radiation is the amplification mechanism of the TWT and CFA.
2) Transition radiation occurs when electrons move with uniform velocity in a spatially inhomogeneous medium, e.g., when electrons pass through perturbations in the medium such as conducting grids, plates, or gaps between conducting surfaces. The klystron exemplifies device operation based on transition radiation.

3) Bremsstrahlung occurs when electrons propagate in a structure where they are subjected to a periodic force created by external electric and/or magnetic fields. Electron cyclotron masers (gyrotrons) and FELs are based on bremsstrahlung.

To generate coherent radiation, the electrons must maintain synchronism with the electromagnetic field. The synchronous resonance condition, which assures the effectiveness of the beam-wave interaction, is expressed as $\omega-k_{z} v_{z}=n \Omega$, where $\omega$ is the wave angular frequency, $k_{z} v_{z}$ is the Doppler shift due to the electron motion with respect to the electromagnetic wave, $k_{z}$ is the axial wavenumber of the electromagnetic field, $v_{z}$ is the electron axial velocity, $\Omega$ is the angular frequency of the electron oscillations due to external (usually magnetostatic or electrostatic) fields, and $n$ is the resonant harmonic number. If $\Omega$ is much different than $\omega$, the synchronous condition requires that the phase velocity of the wave $v_{\mathrm{ph}}=\omega / k_{z}$ be close to the electron velocity $v_{\mathrm{ph}} \approx v_{z}$. This condition is satisfied in amplifiers based on a Cerenkov interaction, but the electromagnetic wave must be slowed down using complex metallic-ceramic periodic circuits, such as helices, coupled cavities, or other RF structures. So-called "slow-wave" amplifiers are based on such circuits. When the electron oscillations are due to a periodic static magnetic field (such as the "wiggler" in an FEL) and $v_{\mathrm{ph}} \approx c$, where $c$ is the speed of light, the synchronous condition is satisfied without slowing the electromagnetic wave. So-called "fast-wave" amplifiers operate under this condition. In gyro-amplifiers, a fast-wave device, the electrons oscillate in a strong homogeneous paraxial magnetostatic field. In gyro-devices operating close to waveguide cutoff, the synchronous condition is given by

$$
\omega \approx \frac{n e B}{\left(m_{0} \gamma\right)}
$$

where $e$ is the electron charge, $m_{0}$ is the electron rest mass, $B$ is the external magnetic field and $\gamma$ is the relativistic factor, $\gamma=1+e V_{b} /\left(m_{o} c^{2}\right)$ expressed in terms of the beam voltage $V_{b}$. In FELs, the electrons oscillate in the presence of a periodic transverse wiggler field. For frequencies well above the waveguide cutoff, the synchronous condition is given by

$$
\omega \approx v_{z}\left(l+\frac{v_{z}}{c}\right) \gamma_{z}^{2}\left(\frac{2 \pi}{l_{w}}\right)
$$

where $v_{z}$ is the axial component of the electron velocity in the presence of the wiggler, $l_{w}$ is the wiggler period, and $\gamma_{z}$ is the relativistic factor associated with the axial electron velocity, i.e., $\gamma_{z}=1 /\left(1-v_{z}^{2} / c^{2}\right)^{1 / 2}$. Thus, one sees a quadratic dependence of the frequency on the electron streaming energy.

The generation of coherent radiation requires that, in addition to the synchronous condition, a condition for electron bunching 
also be satisfied. Bunching may occur if the electron transit time through the RF circuit is longer than the wave period so that one group of electrons may be decelerated by the oscillating electric field, while another group entering the circuit slightly later may be accelerated. It is sometimes fruitful to interpret the bunching mechanism as stimulated emission, which is the classical analog to quantum amplification [2]. The device will produce coherent or "stimulated" emission when the interaction parameters are set so that more electrons are decelerated than accelerated in the high-frequency electric field.

Devices are often classified as either $M$ - or $O$-type according to the type of electron bunching mechanism employed. If bunching occurs by modulating electron positions, typically by using crossed external electric and magnetic fields, the bunching mechanism is $M$-type. Examples are the magnetron and the crossed-field amplifier. If the bunching occurs by modulating electron momentum, through the action of the high-frequency electric field, the bunching process is $O$-type. Examples are klystrons, TWTs, gyro-devices, and FELs. For klystrons and TWTs, the electric-field component parallel to the motion of the electron beam plays the dominant role in the bunching process, while in the case of the gyro-device, the electric-field component perpendicular to the motion of the electron beam has the dominant role. $O$-type devices operate in a linear beam format where the electron streaming velocity $v_{z}$ is parallel to applied magnetic field. In this format, the functions of beam formation, beam-wave interaction, and beam collection are separated, thereby allowing each to be optimized independently.

\section{PeRformance TRENDS}

The history of RF vacuum electronic devices can be viewed as the evolution of conceptual and engineering innovations to satisfy the conditions of synchronism and bunching simultaneously over a wide range of frequencies, thereby generating or amplifying coherent radiation with high peak and average powers. The broad sweep of progress in RF vacuum electronics across a variety of device types is illustrated in terms of the evolution of a "power density" figure-of-merit $P_{\text {av }} f^{2}$, as shown in Fig. 1 [3]. The physical significance of $P_{\mathrm{av}} f^{2}$ derives from the fact that the maximum charge carrier power that can be transported through a RF structure is proportional to its cross-sectional area, or inversely proportional to the square of the operating frequency [4]. In general, the "power density" profile for each amplifier type exhibits the characteristic S-shape curve associated with technological innovation and maturation. As the fundamental limits of a particular device type are approached, progress becomes more difficult and the curve flattens. Escape from the limits imposed by a particular device concept requires finding a better approach. The movement upward and to the right-hand side in the Fig. 1 has been sustained by the introduction of new concepts and improved technology. First, the gridded tube is overtaken by the magnetron under the impetus of wartime demands. The high-power linear-beam tubes, the klystron, and the coupled-cavity TWT then move to the fore in response to accelerator and radar interests. The power limitations of slow-wave devices, imposed by transverse circuit di-

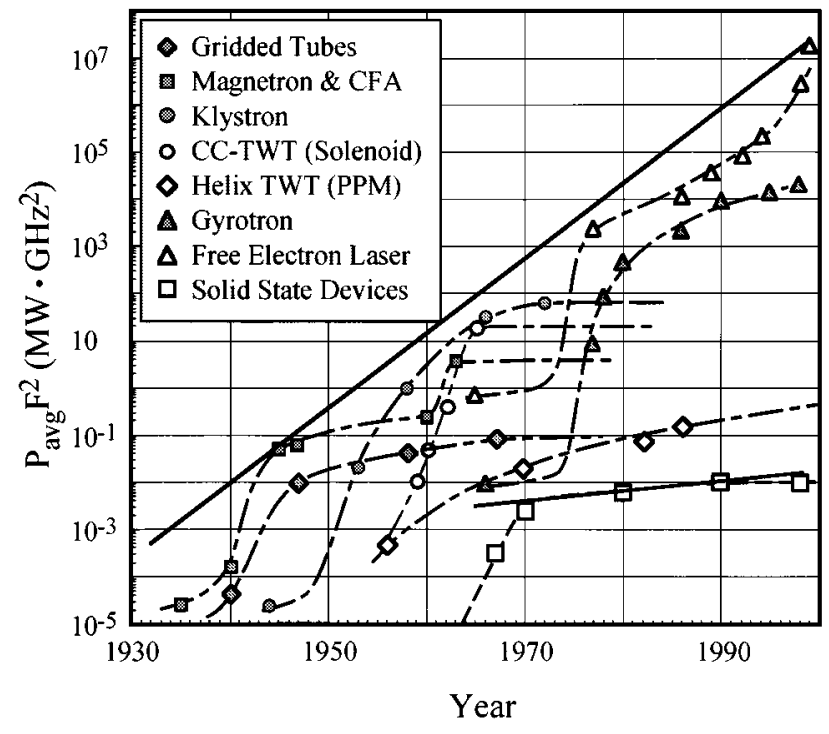

Fig. 1. $P f^{2}$ versus time, showing the evolution of vacuum electronics technology through multiple device generations.

mensions that scale with wavelength, are relieved in fast-wave devices because the interaction mechanism invokes an inherent electron oscillation that can be preferentially matched to the frequency of the operating mode. This being the case, fast-wave devices can employ larger diameter cavities and waveguides that operate in higher order or even optical modes. With this advantage, the gyro-oscillator (gyrotron) and FEL surpass the performance of slow-wave devices in the mid-1970s. Finally, note that a straight line defining a doubling in power density every two years captures the performance envelope for all device types and that this rate of progress has been sustained for 60 years. Progress in RF vacuum electronics, indeed, does continue to evolve at a revolutionary pace.

This portrayal of device performance in terms of power density places a strong emphasis on average power production at higher frequencies and illustrates one primary thrust in vacuum electronics research and development. In some low-duty-cycle applications such as directed energy and particle accelerators, however, the determinant performance parameter is peak power. The current state of technology for generating peak power is shown in Fig. 2 where a distinction is drawn between peak power achieved with pulse duration greater than and shorter than approximately $1 \mu \mathrm{s}$. This distinction serves to highlight a unique performance trait associated with ultrahigh-power sources based on IREB technology [5]. Extremely high peak-power electron-beam accelerators take advantage of explosive electron emission to obtain the desired high-current pulses. This emission process depends on the rapid formation of cathode plasma created by the explosion of microprotrusions on the cathode surface after the application of the high-voltage pulse [6]. Since this plasma expands with a velocity of a few centimeters/microsecond, the small anode-cathode separations (millimeter to centimeter) associated with these high-perveance electron-gun designs become short circuited on a time scale on the order of a microsecond. Explosive electron emission, therefore, is well matched to short pulse $(\tau<1 \mu \mathrm{s})$ operation, but poses a challenge to long-pulse or repetitively pulsed oper- 


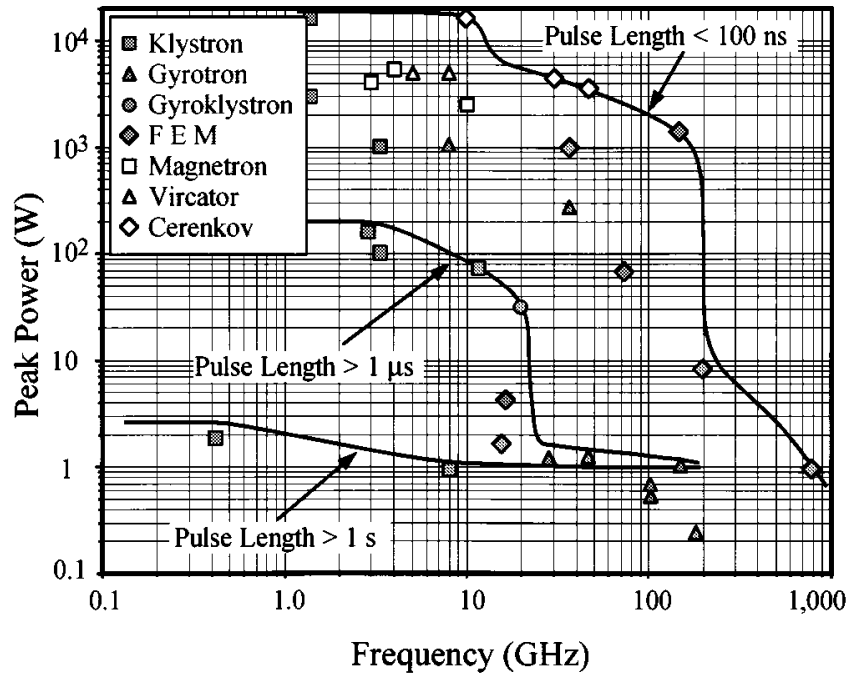

Fig. 2. Device performance in terms of peak power versus frequency. The limits of performance are portrayed for three pulselengths to emphasize constraints imposed by breakdown $(\tau<0.1 \mu \mathrm{s}$ and $\tau>1 \mu \mathrm{s})$ and thermomechanical $(\tau>1 \mathrm{~s})$ issues.

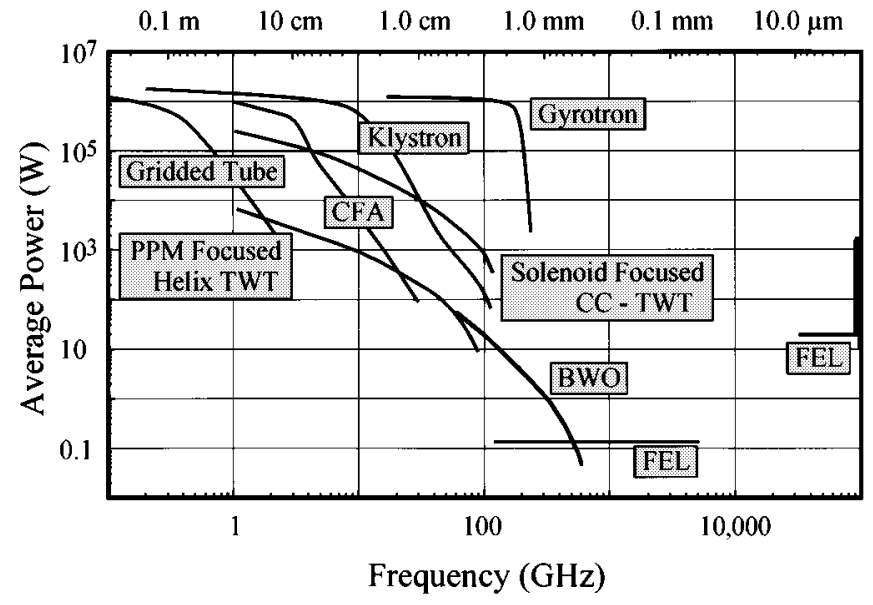

Fig. 3. Present state of vacuum electronics in terms of the ability of multiple device types to generate average power at frequency.

ation. The thermionic cathodes used in longer pulse devices do not suffer this problem, but cannot provide the current densities $\left(\mathrm{kA} / \mathrm{cm}^{2}\right.$ to $\left.\mathrm{MA} / \mathrm{cm}^{2}\right)$ required for ultrahigh power production. Hence, the pursuit of ultrahigh peak power leads inexorably to an increasing constraint on available average power.

Most military, commercial, and scientific applications require power in combination with enhanced bandwidth, efficiency, and fidelity of signal. Vacuum electronics responds to this diversity of requirements with a full spectrum of device types, each offering a unique balance of performance. The current state of technology for the principal device types is shown in Fig. 3 in terms of average power with frequency. Since much of the market exists behind the frontier of available power with frequency, demands have been met by continued innovation in established device types through the implementation of improved design tools and materials technology, as well as, the exploitation of new device concepts. Although these steps may be evolutionary, the impact can be dramatic and important advances continue to be achieved within this broader view of performance.
The TWT best exemplifies the opportunity for continuing strong improvement in established device types. Its robustness is not surprising given its dominance in the market place and user requirements for improvement. As measured in terms of the basic parameters of information theory, the capabilities of the helix TWT are extraordinary. The TWT is a key building block in the rapidly expanding telecommunication systems and retains a major role in satellite-based transmitters. Moreover, with their increasing demand for bandwidth, military radar, electronic countermeasures, and communication systems depend heavily on TWTs. In a package weighing approximately $1 \mathrm{~kg}$, the modern PPM focused TWT provides average powers from tens to a few thousands of watts combined with a gain of $\leq 60 \mathrm{~dB}$ and an instantaneous bandwidth well over an octave. The basic distortion parameters can satisfy the most exacting requirements and the overall efficiency can greater than $70 \%$.

The record of the performance growth achieved with the helix TWT over the past 20 years is outstanding. The instantaneous bandwidth (expressed in terms of the frequency ratio $\left.f_{\max } / f_{\min }\right)$ has been extended nearly threefold from $8 / 2.5$ to $18 / 2$, while the efficiency of wide-band and narrow-band TWTs has nearly doubled to $\sim 50 \%$ and $\sim 70 \%$, respectively. Across this span, the weight of space-based $K u$-band TWTs has been halved to $\sim 0.75 \mathrm{~kg}$, the power increased more than sixfold to $\sim 170 \mathrm{~W}$ and the cost reduced threefold.

With the implementation of a miniaturized reduced gain variant of the PPM focused helix TWT as the vacuum power booster (VPB) in an MPM, the power density of a traveling-wave tube amplifier (TWTA) has been increased nearly tenfold to $\sim 0.7 \mathrm{~W} / \mathrm{cm}^{3}$. Moreover, continuing improvements in power supply and modulator technologies, together with improved amplifier designs, have led to significant improvement in the noise performance of TWTAs. A 200-W X-band MPM, under continuous operation, exhibited a transmitter phase noise of $-133 \mathrm{dBc} / \mathrm{Hz}$ and $-143 \mathrm{dBc} / \mathrm{Hz}$ at offsets of 1 and $10 \mathrm{kHz}$, respectively.

In addition, noteworthy improvements in vacuum amplifier reliability have taken place over the past several decades. The operational lifetimes of the principal types of vacuum electronic amplifiers, always substantial, are now remarkable (see Fig. 4). For demanding space-based applications, service lifetimes must exceed 18 years $(>150000 \mathrm{~h})$. Current data for space-based TWTAs (the full amplifier including TWT, driver. and power conditioning and control circuitry) show a mean-time-to-failure (MTTF) of 18-million h. The reliability improvements of selected amplifiers have led to significant reductions in operating cost for many systems. For example, the cost per operating hour per socket for one of the Navy's high-power radar systems was reduced from $\$ 7.62$ to $\$ 0.36$, a twentyfold reduction within the last decade [7].

\section{Highlights of ReCEnt Progress}

An enormous literature on RF vacuum electronics has been generated over the past 50 years. Development prior to the early 1970s is described in a series of papers in the PROCEEDINGS OF THE IEEE [8]. Many articles on various aspects of the field can be found in an ongoing series of special issues in the IEEE 


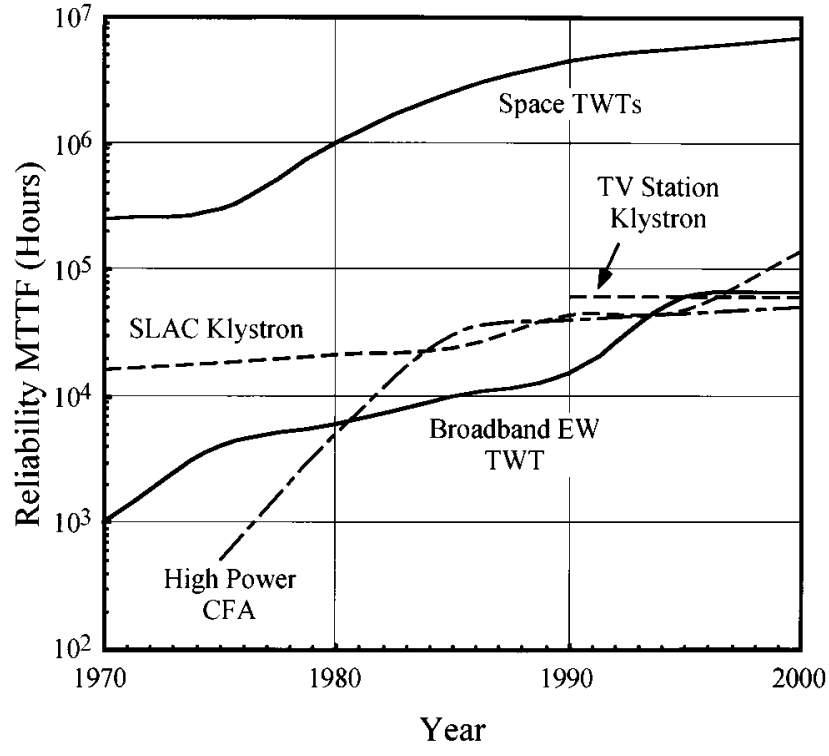

Fig. 4. Advances in reliability for space TWT data (courtesy of J. Christensen BSS Inc.), TV station klystrons (courtesy of R. Symons, Litton ED, a subsidiary of the Northrop Grumman Corporation), accelerator klystrons (courtesy of G. Caryotakis, SLAC), EW TWTs (courtesy of T. Webb, CPI Wireless Solutions), and CFAs (courtesy of J. Dutkowski, NSWC/Crane Division).

TRANSACTIONS ON PLASMA SCIENCE, beginning in 1985 (e.g., see [9]). More recently, a series of comprehensive review papers appeared in a special issue of the PROCEEDINGS OF THE IEEE [10], summarizing both the current status of the field and projections for the future. A special issue of the IEEE TRANSACTIONS ON ELECTRON DEVICES collected many of the most recent advances [11] and an informative article for the layman appeared in the IEEE Microwave Magazine [12]. Finally, many of the high-power sources shown in Fig. 2 are discussed in an excellent review paper in the Review of Scientific Instruments [13].

This section will explore noteworthy advances in the TWT and klystron, as well as more recent innovations such as the MPM, inductive output amplifier (IOA), fast-wave devices, ultra-high-power sources, and RF vacuum microelectronics. These advances can be credited to a combination of device innovation, enhanced understanding gained through improved modeling and design, and the introduction of superior materials [14] and cathodes [15]. Of these supportive technologies, the development of advanced physics-based design codes warrants particular emphasis given the breadth of impact conveyed by this enhanced research and design capability [16].

\section{A. TWTs}

In the design of high-power transmitters for airborne, space, and mobile applications, a strong emphasis is placed on minimizing the power consumption, applied voltage, size, and weight of the amplifiers. For such applications, where instantaneous bandwidth is also a requirement, the helix and coupled-cavity TWTs are the device types of choice. Recent thrusts in TWT development respond to requirements in three primary areas of application. The needs of high-data-rate communication place a strong emphasis on significantly improved fidelity and efficiency. Exploitation of the MPM requires miniaturized highly efficient low-gain VPBs. Also, market demand continues for millimeter-wave TWTs featuring enhanced performance, improved reliability, and lower cost.

1) Helix TWTs: High-data-rate communication demands more efficient use of the spectrum and better transmission quality, which leads inexorably to the use of digital modulation techniques. Additionally, the need for greater RF channel capacity drives systems to increased output power and amplification bandwidth, with some terrestrial applications requiring bandwidth from several hundred megahertz to a few gigahertz. For example, multichannel power amplifiers (MCPAs) used in personal communication systems, local area networks, digital audio radio, and mobile digital (in bands extending from 1.5 to above $2.3 \mathrm{GHz}$ ) require average and peak powers of several hundreds of watts to several kilowatts, respectively.

To obtain the linearity required in the MCPA, the TWT is operated significantly below saturation, which has the effect of reducing its efficiency. However, this effect can be mitigated by designing the helix circuit for reduced gain compression and phase shift of the output signal as the drive level is increased, as well as by using an optimized multiple-stage depressed collector (MSDC). This approach, enabled by accurate design codes, has produced outstanding TWT performance at $L$ - and $S$-bands. For example, an $L$-band TWT, with an eight-tone intermodulation level for continuous random phasing of the signals of $-26 \mathrm{dBc}$, has been designed to provide over $250 \mathrm{~W}(\mathrm{CW})$ at 9-dB backoff from saturation $(2 \mathrm{~kW})$ with an efficiency greater than $25 \%$. The $S$-band TWT provides $500 \mathrm{~W}(\mathrm{CW})$ at $6 \mathrm{~dB}$ backoff from saturation $(2.05 \mathrm{~kW})$ with an efficiency of $40 \%$. MCPAs using these TWTs have produced eight-tone C/3IM levels of $-70 \mathrm{dBc}$ at 200-300-W outputs, which is nearly a fourfold improvement over solid-state systems with the same fidelity and twice the efficiency [17].

Similar approaches are expected to yield these same benefits in the space-TWT field, albeit at higher frequencies. The current state of space-qualifiable millimeter-wave TWT technology is exemplified by a $Q$-band TWT operating at $44.5 \mathrm{GHz}$ with a continuous power of $160 \mathrm{~W}$. The TWT exhibits excellent gain flatness (better than $1.5 \mathrm{~dB}$ ) over the specified 2-GHz bandwidth and has a $3-\mathrm{dB}$ instantaneous bandwidth of $10 \mathrm{GHz}$. At this time, a two-stage depressed collector is used to obtain an efficiency of $35 \%$; the use of more stages can increase this to standard space-TWT values [18].

The MPM evolved from the belief that amplifier miniaturization would prove attractive for weight- and size-constrained systems requiring high efficiency. The MPM synergistically combined evolutionary improvements in vacuum and solid-state technology; by partitioning the gain in the amplifier chain, the inherent efficiency of the VPB (a low-gain TWT) and the low-noise characteristics of the solid-state driver (a broad-band MMIC amplifier) were captured in a single building-block RF module.

The highlight of early MPM development was the demonstration of a 100-W (CW) transmit module, operating from 6 to $18 \mathrm{GHz}$ with $45 \%$ band-center efficiency and a noise figure of $\sim 10 \mathrm{~dB}$ [19]. This $120-\mathrm{cm}^{3}$ fluid-cooled unit, which weighed $363 \mathrm{~g}$, offered a fourfold improvement in efficiency over the MMIC alone, a greater than 20-dB improvement in noise figure over the TWT alone, and a greater than tenfold reduction in size 
relative to a TWTA or SSPA. The dramatic breakthrough in microwave transmitter packaging, efficiency, and broad-band performance represented by this technology are extremely attractive for a wide range of applications.

MPM technology continues to evolve in many directions. A $C$-band VPB has been developed that provides $\mathrm{CW}$ power over an octave bandwidth in excess of $170 \mathrm{~W}$ with an overall efficiency of $65 \%$ [20]. The MPM efficiency from dc power in to RF power out is $>50 \%$. MPM bandwidth has been extended beyond three octaves, with CW powers of $100 \mathrm{~W}$ over $2-18 \mathrm{GHz}$. The MPM format also supports active electronically steered arrays up to $X$-band, with half-wavelength cross-sectional areas having been developed up to $12 \mathrm{GHz}$ with $120 \mathrm{~W}$ (peak) at $20 \%$ duty.

2) Coupled Cavity TWTs: The CC-TWT uses a slow-wave circuit that is mechanically and thermally more robust than a helix. At millimeter wavelengths, the low-frequency design approach of stacking an assembly of round staggered-slot cavities is extremely expensive (small high-tolerance parts and stack assembly along with low yield of final assembly). Several approaches have been taken to overcome these fabrication problems. Of these, the ladder-core structures have been most successful [21]. This approach first uses a wire EDM process to cut a complete section of cavities in a copper block and then cover plates are added to close off the circuit. Ladder-circuit TWTs have been built that operate at powers ranging from $50 \mathrm{~W}$ to $50 \mathrm{~kW}$, at frequencies from 30 to $95 \mathrm{GHz}$ with bandwidths from $1 \%$ to $20 \%$. An initial demonstration recently has been completed of a $980-\mathrm{W}$ peak-power amplifier operating at $96.5 \mathrm{GHz}$ with an instantaneous bandwidth of $3.0 \mathrm{GHz}$. The $W$-band CC-TWT features a gain of $\sim 47 \mathrm{~dB}$ with an efficiency of $6.4 \%$ and can be operated at duty factor of $10 \%$ duty [22]. Current interest in this capability focuses on opportunities in airborne imaging radar. Looking toward the future, "deep etch" or lithographie galvanoformung abformung (LIGA) microfabrication techniques may provide a more accurate and less expensive alternative for the construction of such circuits at millimeter-wave and higher frequencies.

\section{B. Klystrons}

Klystrons are efficient high-gain amplifiers that can be designed to operate at frequencies from the UHF to the millimeterwave. These amplifiers require a relatively high voltage and operate over narrow bandwidths (1\%) at low power to moderate bandwidths $(10 \%)$ at higher power. For a typical lownoise klystron, phase noise is on the order of $-145 \mathrm{dBc} / \mathrm{Hz}$ at $1 \mathrm{kHz}$ from the carrier and the noise figure is $\sim 35 \mathrm{~dB}$. For many applications, the klystron is constrained by high operating voltage, limited bandwidth, and rapidly declining power at high frequency.

1) Clustered Cavity Klystron: In the clustered cavity klystron, the technique of grouping cavities that had been previously used in extended interaction output circuits was extended to the prior cavities [23]. In this case, however, the cavities within a group were not mutually coupled. With this technique, the clustered cavity klystron has demonstrated bandwidths up to $20 \%$. This approach has been exploited by developing a wide-band klystron that can replace two narrower band amplifiers operating in separate, but adjacent channels, thus allowing one tube to cover the entire frequency band while holding the other as an online spare.

2) Multiple Beam Klystron (MBK): The multiple-beam concept has long been recognized as offering the potential for reduced voltage, size and weight, and increased efficiency and bandwidth. The success of the 20-year Russian effort derives from their focus on fundamental-mode resonator designs. As recently reported [24], a family of pulsed and CW MBKs has been developed. Pulsed MBKs with peak power in the tens of kilowatt and frequencies in the approximate range of $6-15 \mathrm{GHz}$ operate with bandwidths of $\sim 6 \%$. With bandwidths comparable to CC-TWTs, these MBKs offer higher efficiency $(\sim 40 \%)$ and lower phase sensitivity. Compared to conventional single-beam klystrons, these MBKs, incorporating a single-gap output cavity design, provide a twofold to two-and-one-half-fold increase in bandwidth. To cite an extreme example, an $18 \%$ bandwidth was obtained in a low-frequency $(<3 \mathrm{GHz}) 6-\mathrm{kW}$ amplifier incorporating 61 beams. Major technical issues with the MBK concern the requirement for relatively high cathode current density $\left(>10 \mathrm{~A} / \mathrm{cm}^{2}\right)$ and the complexity of design and, hence, of manufacturing.

3) Extended Interaction Klystron: The continuing progress in the development of millimeter-wave extended interaction klystrons provides another highlight for this device type. An initial demonstration recently has been completed of a very compact $1.0-\mathrm{kW}$ peak-power amplifier operating at $94.5 \mathrm{GHz}$ with an instantaneous bandwidth of $0.9 \mathrm{GHz}$. This $W$-band klystron, featuring a gain of $47 \mathrm{~dB}$, an efficiency of $8 \%$, and a duty factor of $10 \%$, is intended for application in airborne imaging radar [25].

4) Klystrons for Linear Colliders: Increasing the RF linac drive frequency can decrease the overall length and cost of a high-energy electron-positron collider. Hence, a major thrust in accelerator technology has been the development of a highpower $X$-band klystron operating at $11.424 \mathrm{GHz}$. The current status of this effort is defined by a 75-MW peak-power solenoid-focused klystron with an efficiency of 50\%. Associated with this power level is an applied voltage of $450 \mathrm{kV}$ and pulse duration of $1.2 \mu \mathrm{s}$. Work on a 60-75 MW PPM focused version is nearly complete [26].

\section{IOAs}

The IOA is an intriguing device concept that combines the beam modulation attributes of gridded tubes with the electron beam acceleration and power extraction circuitry of linear-beam tubes. The klystrode, a modern version of the inductive output tube, was developed for television broadcast [27]. The klystrode uses a cavity-driven cathode-grid region to form a modulated electron stream that is then post-accelerated in a high-voltage electron gun. The pre-bunched beam is injected into a short beam tunnel after which it passes through the gap of a klystron-like output cavity. The advantages obtained by injecting a pre-bunched beam are high efficiency and a significant reduction in the required RF interaction length; i.e., the single cavity of a klystrode replaces the many cavities of 
a klystron. The reduced length of the klystrode simplifies the beam transport magnetics, thereby, reducing the weight. Despite its advantages in power density and efficiency, the range of klystrode applicability is limited by the severe constraints in gain-bandwidth product imposed by the transconductance of the thermionic cathode-grid configuration. Nevertheless, the klystrode is highly competitive in such very narrow-band UHF applications as television transmission and the excitation of linear accelerators. More recently, a multistage-depressed collector was added to the klystrode, yielding a device capable of amplifying signals of widely varying amplitude with almost constant efficiency. This constant efficiency amplifier offers distinct advantage for waveforms such as that selected for digital broadcast television [28].

\section{Gyro-Devices}

The cyclotron resonance interaction can be implemented in oscillator and amplifier configurations analogous to those of slow-wave devices. The gyro-oscillator, or gyrotron, is a singlecavity device that can generate unprecedented average and peak power levels at millimeter wavelengths with high efficiency. The key to such high-power operation is the ability to operate on higher order cavity modes. Gyro-amplifiers have been developed using klystron-, twystron-, and TWT-like interaction circuits. In the gyro-klystron, azimuthal bunching is achieved by passing the beam through several cavities separated by beam tunnels before being injected into a final output cavity designed for power extraction. Wider bandwidth can be obtained by using a uniform or tapered waveguide for bunching and power extraction (gyro-TWT) or by following an initial bunching cavity with a waveguide circuit (gyro-twystron).

1) Gyrotrons (Gyro-Oscillators): The primary applications for gyrotrons are the cyclotron resonance heating of fusion plasmas, ceramic sintering, and metal joining. Representative of the current state of gyrotron technology is a $110-\mathrm{GHz}$ gyrotron oscillator capable of operation at $1 \mathrm{MW}$ and an efficiency of $31 \%$ for 5 -s pulses and at $400 \mathrm{~kW}$ and $30 \%$ efficiency for $10-\mathrm{s}$ pulses. To achieve these unprecedented powers without thermal damage to the interaction circuit, this device was designed to operate in the $\mathrm{TE}_{22,6,1}$ cavity mode. Given the high order of this mode, these gyrotrons incorporate internal mode converters that convert the TE mode to a more usable output mode such as a TEM $_{00}$ Gaussian beam. Gyrotrons have been developed, or are under development, to operate at frequencies ranging from 8 to $170 \mathrm{GHz}$. Lower power gyrotrons intended for scientific applications have been developed at frequencies as high as $800 \mathrm{GHz}$ [29].

2) Gyro-Amplifiers: Gyro-klystrons have been developed in several frequency bands for accelerator and radar applications. Gyro-klystrons designed for driving RF linear accelerators must provide very high peak powers, but at low duty cycle with narrow instantaneous bandwidth. Alternatively, radar operation demands both average and peak power and places a strong emphasis on bandwidth. Examples of achieved performance include an experimental amplifier designed for accelerator application that operated in fundamental mode and yielded a peak power of $27 \mathrm{MW}$ at $9.87 \mathrm{GHz}$. A subsequent second harmonic device achieved a peak power of $32 \mathrm{MW}$ at $19.76 \mathrm{GHz}$ [30].
Comparable power gyro-klystrons at even higher frequency (30 and $91 \mathrm{GHz}$ ) are under study.

The need for atmospheric transmission focuses radar interest development at $\sim 35 \mathrm{GHz}$ and $\sim 94 \mathrm{GHz}$. Recent efforts include a $90-\mathrm{kW}$ peak-power $10-\mathrm{kW}$ average-power gyro-klystron amplifier developed for operation at $94 \mathrm{GHz}$ for radar experimentation. The $W$-band amplifier, driven by a 65-kV 6-A electron beam, has an instantaneous bandwidth of $600 \mathrm{MHz}$ and an efficiency of 33\% [31]. This level of performance represents a fourfold increase in gyro-klystron average power at $W$-band and a twentyfold improvement over that available from a CC-TWT. In addition to power and bandwidth, the fidelity of the signal is equally important to modern radar development. Noise measurements with a $35-\mathrm{GHz} 200-\mathrm{kW}$ gyro-klystron have shown the single-sideband phase noise to be $-147 \mathrm{dBc} / \mathrm{Hz}$ at $10-\mathrm{MHz}$ offset from the carrier [32], a level comparable to that of a conventional high-power klystron. For those applications where still greater bandwidth is required, the gyro-twystron and gyro-TWT offer progressively increasing capability, albeit with greater challenge in achieving amplifier stability. At the extremes of gain and bandwidth, millimeter-wave gyro-TWTs have demonstrated gains as high as $70 \mathrm{~dB}$ [33] and bandwidths as large as 30\% [34].

\section{E. FELs}

The inverse scaling of wavelength with electron energy [see (2)] allows the FEL to produce very high-frequency radiation when either high-energy beams or short-period wigglers are used. In practical applications, however, high gain requires high wiggler field amplitudes, which, in turn, precludes very short wiggler periods; hence, high-frequency operation can entail relatively high beam energies. This often precludes the use of standard power supplies and modulators, requiring instead relatively large and complex electron accelerators. As a result, FELs are principally useful when infrared or shorter wavelengths are needed [35].

Most existing FELs operate in the infrared where the high-intensity picosecond pulses they produce are used in materials and biomedical research. At shorter wavelengths, FELs have been used to make measurements of time-resolved fluorescence decays of biological molecules to study time-resolved photoemission and photo-ionization.

Higher average power and ultrashort wavelengths are two primary areas of future development for FEL technology. The goal at infrared or shorter wavelengths is several tens of kilowatts of average power and the most likely configuration is an oscillator driven by an RF linear accelerator. The highest level of achieved performance is an average power of $1.7 \mathrm{~kW}$ at a wavelength of $3 \mu \mathrm{m}$ and upgrades are underway to extend the performance to $10 \mathrm{~kW}$ [36].

Short-wavelength FELs primarily directed toward research applications of X-rays at wavelengths down to $1 \AA$. Currently, the principal X-ray sources are synchrotrons, but FELs hold promise for substantial increases in the average brilliance. Current designs call for beam energies of the order of $14 \mathrm{GeV}$, peak beam currents of approximately $3.4 \mathrm{kA}$, and wiggler lengths of approximately $100 \mathrm{~m}$. The construction of such a source is clearly a major financial undertaking. 


\section{F. Ultrahigh-Power Sources}

The development of short-pulse $(10 \mathrm{~ns}<\tau<1.0 \mu \mathrm{s})$ high-voltage electron accelerators in the mid-1960s for nuclear weapon effects simulation provided the capability for producing relativistic electron beams with peak powers in excess of $10 \mathrm{GW}$. Long intrigued by the advantages of working with relativistic electrons for microwave power production, the availability of the IREBs made possible by this technology stimulated researchers to undertake an ongoing effort in the generation of coherent electromagnetic radiation. These pulse-line accelerators have been used to study a wide variety of relativistic device concepts, many of which are intense beam extensions of the more established device types [13], [37]. In such devices as the relativistic magnetron, Cerenkov (rippled wall) TWTs and BWOs, relativistic gyro-devices and free electron maser (FEM), the self-field effects associated with the use of an IREB are detrimental to device performance and, therefore, represent challenges to be overcome by innovative design. Of greater interest are those concepts such as the Vircator (the IREB equivalent to the reflex klystron), relativistic klystron amplifier/oscillator, the Reltron, and the magnetically insulated line oscillator (MILO) that use the self-field effects as an inherent element of device operation. The Reltron and MILO exemplify this exploitation of high space charge and self-magnetic fields.

1) Reltron: The Reltron is a relativistic variant of the klystrode in which a virtual cathode acting in a resonant cavity is used to form a modulated electron beam that is then post-accelerated into a multicavity circuit for power extraction. Reltron performance is typified by an $L$-band device that produced $600 \mathrm{MW}$ at $1 \mathrm{GHz}$ with a pulse duration $\leq 1.0 \mu \mathrm{s}$ and an efficiency of $40 \%$. The beam parameters were as follows: injector voltage $-250 \mathrm{kV}$, beam current $-1.35 \mathrm{kA}$, and post-acceleration voltage $-750 \mathrm{kV}$ [38]. Reltrons have been designed for operation over a frequency range of $0.5-6 \mathrm{GHz}$ with tuning ranges up to $30 \%$. A duty factor of $10^{-5}$ has been demonstrated, giving average powers of greater than $4 \mathrm{~kW}$.

2) MILO: The MILO is a low-impedance intense-beam crossed-field oscillator that exploits the self-magnetic field associated with the electron flow rather than requiring an external magnet. The MILO configuration consists of a coaxial transmission line formed by a central cathode stalk that extends axially into a periodic slow-wave structure formed by a series of azimuthally symmetric anode vanes extending radially inward from the outer wall. With the application of a fast-rise-time negative-voltage pulse, the explosive electron emission originating along the outer surface of the cylindrical cathode is radially confined by the self-magnetic field so that the electrons are forced to flow parallel to the axis. The electrons undergo cross-field drift with some striking the grounded slow-wave structure. RF power is extracted from the downstream elements of the circuit. MILO performance is typified by peak powers greater than $1 \mathrm{GW}$ at frequencies near $1 \mathrm{GHz}$ and efficiencies of approximately $5 \%$ with applied voltages of approximately $500 \mathrm{kV}$ [36], [39].

\section{G. Modeling and Simulation}

The process of microwave power tube development has been characterized historically by a time-consuming cycle of first- order design (using older generation design codes), cold test of a circuit test vehicle, redesign, device fabrication, and hot test. Acknowledging the rapid evolution of computer hardware and software, an effort has been underway to develop an interactive suite of multidimensional physics-based computational design tools for the full range of RF vacuum devices [16]. These codes are intended to break this time-tested cycle, thereby resulting in significant reduction in device development time and cost, improved device performance (as measured in reliability as well as the usual attributes of power, bandwidth, efficiency, etc.), and enhanced physical insight leading to next-generation device concepts. Inherent in this thrust is the objective of achieving "first pass design success." In other words, the intent is to establish a design capability of sufficient integrity and validity that computational design and optimization will yield a prototype meeting the designed specifications in its first embodiment.

The ability to design vacuum electronic amplifiers using simulation tools has had impact across all amplifier types, including crossed-field amplifiers, gyro-devices, klystrons, and TWTs. Two examples serve to highlight the impact of this capability and provide a measure of recent progress. The highly successful $W$-band gyro-klystron, described above, was designed and optimized in only two steps using a suite of recently completed codes. The initial high peak-power low-duty prototype yielded a measured performance in its first testing that matched predicted values in all aspects. Having established the design protocol, the low-duty design was modified for high-duty operation. The demonstrated performance of this high average-power gyro-klystron again demonstrated "first pass design success."

The recent development of an extremely efficient wide-band VPB for the $C$-band MPM, described above, provides a more meaningful measure of the value of such codes on the TWT development cycle [12]. The first stage of the VPB development had begun before the initial phase of the helix TWT design code suite became available. Using the traditional design approach, extension of the VPB electronic efficiency from $15 \%$ to $33 \%$ had required the design, modification, and testing of 14 prototypes over two years. Once the first of the advanced codes became available, the electronic efficiency was extended to $42 \%$ in a single step requiring less than two months. This design process began with the use of a one-dimensional (1-D) code to optimize the helix pitch for maximum efficiency. A module in the code carried out the 12-parameter optimization and provided the pitch profile yielding maximum efficiency. The TWT was then built using the computed pitch profile and tested to demonstrate the efficiency. This initial design, derived from the 1-D code, suffered beam interception on the helix, reducing the total amplifier efficiency. Using the multidimensional version of the code, the design of the $C$-band VPB was refined still further. By tracking particles in the multidimensional simulation, the location on the helix of the beam interception was determined; the intercept current was then eliminated by adjusting the strength of the magnetic field in that location. Using this methodology, a total efficiency of $65 \%$ was achieved for the VPB by using a four-stage depressed collector. In this development, excellent agreement was observed between predicted and measured values of performance [40]. Due to the number 
of parameters adjusted in this optimization process, achieving this level of performance in the laboratory using trial-and-error methods would have been arduous and costly, if not impractical.

\section{H. Vacuum Microelectronics}

RF vacuum microelectronics captures the momentum associated with the mainstream pursuit of reduced scale electronics, as well as emergent capabilities for micromachining, and focuses them in an attempt to exploit the recognized advantages of electron propagation in a vacuum. The most advanced vacuum microelectronics technology is based on field emission from an array of sharply pointed microstructures located in near proximity to gate apertures. Electron emission from the negative electron affinity surfaces provided by wide-bandgap semiconductors is a more speculative option [15].

The role of a VPB in an MPM appears well matched to the perceived characteristics of a vacuum microelectronic emitter. The power booster is a low-to-moderate gain device that emphasizes high efficiency and power density. Potential MPM applications extend from frequencies in the UHF to the millimeter wave and include narrow- to wide-band requirements. From the perspective of source development, the combination of narrow-band operation at low frequency and low gain minimize the demand for high transconductance and reduced array capacitance. IOA configurations such as the klystrode and its helix-based analog, the twystrode are well matched to this concept. In these devices, an advanced electron injector (perhaps a gated FEA) would be substituted for the electron emission and beam modulation elements [41]. With this approach, klystrode operation with a several percent bandwidth could be extended into the microwave region by implementing an electron injector based on attainable, high-performance $(10 \mu \mathrm{A} /$ tip, $1 \mu \mathrm{S} /$ tip, $3-\mu \mathrm{m}$ tip spacing, and $1-\mu \mathrm{m}$ insulator thickness) FEAs [42]. Although modulated FEA operation has been extended to $10 \mathrm{GHz}$ and all required performance attributes (current density, lifetime, transconductance, etc.) have been demonstrated individually, significant challenges must be overcome before a practical cathode for modulated emission becomes available [43].

A very intriguing interim step, however, was taken in a recent unmodulated experiment. More specifically, a 1-mm-diameter molybdenum FEA operating at a current density of $11.5 \mathrm{~A} / \mathrm{cm}^{2}$ was used in an unmodulated electron gun to inject a $91-\mathrm{mA}$ $3.5-\mathrm{kV}$ electron beam through a helix circuit into the collector with a beam transmission of $99.5 \%$. Under these conditions, a $\mathrm{RF}$ power of $55 \mathrm{~W}$ was produced at $4.5 \mathrm{GHz}$, corresponding to an electronic efficiency of $17 \%$. Although issues of lifetime have yet to be evaluated and further consideration must be given to FEA protection schemes, this experiment does demonstrate the successful integration of an FEA into a PPM focused TWT [44].

\section{OUTLOOK FOR VACUUM ELECTRONICS}

"Smaller, lighter, and cheaper" are the watchwords for the near-term future of vacuum electronics. Performance cannot be sacrificed to meet these goals-it must be improved! However, in the last decade, the tools to achieve these goals have been de- veloped and their imaginative use is expected to propel vacuum electronics technology in new directions. Several of the following supplementary technologies, already noted, will contribute to this movement.

1) Advances in computer hardware and software will support the development of faster more accurate simulation and modeling capability.

2) The development of better more sophisticated high-voltage technology will increase the reliability and reduce the volume and weight of power supplies.

3) New materials (dielectrics and magnetics) and electron sources will improve performance and reduce costs.

Some currently perceived directions for vacuum electronics technology include the development of low-gain high-power vacuum-electronic devices across the range of device types for use as the final stage of radar, electronic warfare, and communication amplification. For example, a low-gain TWT can be optimized for linearity and efficiency and, when driven by an improved solid-state amplifier, the noise performance of the amplifier chain will be better than that of a comparable output power high-gain vacuum electronic amplifier. This low-gain TWT could be designed for ease of manufacture: it may not require a sever, it may operate with low or no magnetic field, and a more uniform circuit could be ensured. The translation of these attributes to size, weight, and cost savings is apparent. This same approach, however, can be applied to other vacuum electronic device types, such as the MBK.

With increasing demands for data rate and bandwidth, the need for high power at high frequencies will continue to grow. The technical understanding gained from the use of overmoded structures in fast-wave device development can be expanded into the slow-wave area to meet high-frequency power needs in compact devices. The future will also see an extension of bandwidth in fast-wave devices in both the gyro-klystron and gyro-TWT formats for extremely high-power needs.

The dynamic optimization of a vacuum electronic device will support better performance at several in-band frequencies. This concept, now in use with current amplifier technology, will be carried to a higher degree of sophistication using the suite of advanced three-dimensional (3-D) modeling and simulation tools to tailor the performance in regimes of interest.

As noted, the future of vacuum electronics will depend on advances in other technologies. Dielectric materials will be tailored to device performance needs; magnetics will provide beam focussing with less weight. Electron sources will include a full range of new cold emitters, including wide-bandgap semiconductors and laser-pumped photocathodes. Microfabrication techniques (including a mature field-emitter array capability), such as those being developed for microelectromechanical systems (MEMS) and including techniques such as LIGA, will be adapted in the drive toward increasing sophistication in vacuum electronic devices, including the capability for distributed arrays.

\section{REFERENCES}

[1] L. D. Landau and E. M. Lifshitz, "Landau and Lifshitz course of theoretical physics," in Electrodynamics of Continuous Media, 2nd ed. New York: Pergamon, 1984, vol. 8, ch. XIV. 
[2] A. I. Gaponov, M. I. Petelin, and V. K. Yulpatov, "The induced radiation of excited classical oscillators and its in high frequency electronics," Radiophys. Quantum Electron., vol. 10, pp. 794-814, 1967.

[3] V. L. Granatstein, R. K. Parker, and C. M. Armstrong, "Vacuum electronics at the dawn of the twenty-first century," Proc. IEEE, vol. 87, pp. 702-716, May 1999.

[4] L. S. Nergaard, "Tubes and/or solid-state devices for power generation," Microwave J., vol. 13, pp. 65-67, Apr. 1970.

[5] R. B. Miller, An Introduction to the Physics of Intense Charged Particle Beam. New York: Plenum, 1982.

[6] R. K. Parker, R. E. Anderson, and C. V. Duncan, "Plasma-induced field emission and the characteristics of high current relativistic electron flow," J. Appl. Phys., vol. 45, pp. 2463-2479, June 1974.

[7] J. Dutkowski, private communication.

[8] Proc. IEEE, vol. 61, pp. 279-381, Mar. 1973.

[9] IEEE Trans. Plasma Sci., vol. 28, June 2000.

[10] Proc. IEEE, vol. 87, pp. 699-889, May 1999

[11] IEEE Trans. Electron Devices, vol. 48, pp. 1-182, Jan. 2001

[12] R. H. Abrams, B. Levush, A. A. Mondelli, and R. K. Parker, "Vacuum electronics in the 21st century," IEEE Microwave Mag., vol. 2, pp. 61-72, Sept. 2001.

[13] S. H. Gold and G. S. Nusinovich, "Review of high-power microwave source research," Rev. Sci. Instrum., vol. 68, pp. 3945-3974, Nov. 1997.

[14] J. P. Calame and D. K. Abe, "Applications of advanced materials technologies to vacuum electronics," Proc. IEEE, vol. 87, pp. 840-864, Mar. 1999.

[15] J. A. Nation, L. Schachter, F. M. Mako, L. K. Len, W. Peter, C. M. Tang, and T. Srinivasan-Rao, "Advances in cold cathode physics and technology," Proc. IEEE, vol. 87, pp. 865-889, May 1999.

[16] T. M. Antonsen, Jr., A. A. Mondelli, B. Levush, J. P. Verboncoeur, and C. K. Birdsall, "Advances in modeling and simulation of vacuum electronic devices," Proc. IEEE, vol. 87, pp. 804-839, May 1999.

[17] D. M. Goebel, R. R. Liou, W. L. Menninger, X. Zhai, and E. A. Adler, "Development of linear traveling wave tubes for telecommunications applications," IEEE Trans. Electron Device, vol. 48, pp. 74-81, Jan. 2001

[18] D. R. Dibb and R. H. Le Borgne, "45 GHz TWT—Recent advances," in Int. Vac. Electron. Conf., Monterey, CA, 2000.

[19] C. Smith, C. M. Armstrong, and J. Duthie, "The microwave power module-A versatile RF building block for high power transmitters," Proc. IEEE, vol. 87, pp. 717-737, Mar. 1999.

[20] D. R. Whaley, C. M. Armstrong, B. Gannon, G. Groshart, E. Hurt, J. Hutchins, M. Roscoe, and T. M. Antonsen, Jr, "Sixty-percent-efficient miniature $C$-band vacuum power booster for the microwave power module," IEEE Trans. Plasma Sci., vol. 26, pp. 912-921, June 1998.

[21] B. James and P. Kolda, "A ladder circuit coupled cavity TWT at 80-100 GHz," in Int. Electron Devices Meeting Dig., Los Angeles, CA, 1986, pp. 494-497.

[22] B. G. James, private communication.

[23] R. S. Symons, B. Arfin, R. E. Boesenberg, P. E. Ferguson, M. Kirshner, and J. R. M. Vaughn, "An experimental clustered cavity klystron," in Int. Electron Devices Meeting Dig., Washington, DC, 1987, pp. 153-156.

[24] E. A. Gelvich, L. M. Borisov, Y. V. Zhary, A. D. Zakurdayev, A. S. Pobedonostev, and V. I. Poognin, "The new generation of high-power multiple-beam klystrons," IEEE Trans. Microwave Theory Tech., vol. 41, pp. 15-19, Jan. 1993.

[25] B. Steer, private communication

[26] R. M. Phillips and D. W. Sprehn, "High-Power klystrons for the next linear collider," Proc. IEEE, vol. 87, pp. 738-751, Mar. 1999.

[27] D. H. Preist and M. B. Shrader, "The klystrode-An unusual transmitting tube with potential for UHF-TV," Proc. IEEE, vol. 70, pp. 1318-1325, Nov. 1982.

[28] R. Symons, "The constant efficiency amplifier," in Microwave Vacuum Electron Device Con., Monterey, CA, 1998.

[29] K. L. Felch, B. G. Danly, H. R. Jory, K. E. Kreischer, W. Lawson, B. Levush, and R. J. Temkin, "Characteristics and applications of fast-wave gyrodevices," Proc. IEEE, vol. 87, pp. 752-781, Mar. 1999.

[30] V. L. Granatstein and W. Lawson, "Gyro-amplifiers as candidate RF drivers for TeV linear colliders," IEEE Trans. Plasma Sci., vol. 24, pp. 648-665, June 1996.

[31] B. G. Danly, M. Blank, J. P. Calame, B. Levush, K. T. Kguyen, D. E. Pershing, R. K. Parker, K. L. Felch, B. G. James, P. Borchard, P. Cahalan, T. S. Chu, H. R. Jory, T. A. Hargraves, R. B. True, W. G. Lawson, and T. M. Antonsen, "Development and testing of a high-average power 94-GHz gyroklystron," IEEE Trans. Plasma Sci., vol. 28, pp. 713-726, June 2000 .
[32] J. P. Calame, B. G. Danly, M. Garven, and B. Levush, "Studies of electronic noise in gyroklystrons," Phys. Plasmas, vol. 7, pp. 2180-2185, May 2000.

[33] K. R. Chu, H. Y. Chen, C. L. Hung, T. H. Chang, L. R. Barnett, S. H. Chen, and T. T. Yang, "Ultrahigh gain gyrotron traveling wave amplifier," Phys. Rev. Lett., vol. 81, pp. 4760-4763, 1998.

[34] G. S. Park, J. J. Choi, S. Y. Park, C. M. Armstrong, A. K. Ganguly, R. H. Kyser, and R. K. Parker, "Gain broadening of two-stage tapered gyro-TWT amplifier," Phys. Rev. Lett., vol. 74, pp. 2399-2402, Mar. 1995

[35] H. P. Freund and R. K. Parker, "Free electron lasers," Sci. Amer, vol. 260, pp. 84-89, Apr. 1989.

[36] H. P. Freund and G. R. Neil, "Free-electron lasers: Vacuum electronic generators of coherent radiation," Proc. IEEE, vol. 87, pp. 782-803, Mar. 1999.

[37] R. J. Barker and E. Schamiloglu, Eds., High-Power Microwave Sources and Technologies. Piscataway, NJ: IEEE Press, 2001.

[38] R. B. Miller, C. A. Muehlenweg, K. W. Habinger, and J. R. Clifford, "Super-Reltron progress," IEEE Trans. Plasma Sci., vol. 22, pp. 701-705, Oct. 1994.

[39] M. C. Clark, B. M. Marder, and L. D. Bacon, "Magnetically insulated transmission line oscillator," Appl. Phys. Lett., vol. 52, pp. 78-80, Jan. 1988.

[40] D. R. Whaley, K. E. Kreischer, V. O. Heinen, D. Chernin, T. M. Antonsen, Jr., and B. Levush, "CHRISTINE3D modeling and experimental validation," in 2nd IEEE Int. Vac. Electron. Conf., 2001, pp. 161-162.

[41] R. K. Parker, "RF vacuum microelectronics," , vol. 52, pp. 366-370, July-Sept. 1996.

[42] J. P. Calame, H. F. Gray, and J. L. Shaw, "Analysis and design of microwave amplifiers employing field-emitter arrays," J. Appl. Phys., vol. 73, pp. 1485-1504, Feb. 1993.

[43] K. L. Jensen, R. H. Abrams, and R. K. Parker, "Field emitter array development for high frequency applications," J. Vac. Sci. Technol. B, Microelectron. Process. Phenom., vol. 16, pp. 749-753, Mar.-Apr. 1998.

[44] D. R. Whaley, B. Gannon, C. R. Smith, C. A. Armstrong, and C. A. Spindt, "Application of field emitter arrays to microwave power amplifiers," IEEE Trans. Plasma Sci., vol. 28, pp. 727-747, June 2000.

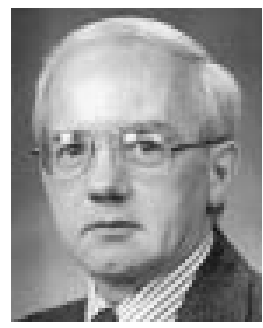

Robert K. Parker (M'85-SM'93-F'95) received the B.S. degree in physics from Allegheny College, Meadville, PA, in 1964, the M.S. degree in space physics engineering from the Air Force Institute of Technology, Wright-Patterson AFB, OH, in 1966, and the Ph.D. degree in nuclear engineering (plasma physics) from the University of New Mexico, Albuquerque, in 1973.

$\mathrm{He}$ is currently the Head of the Vacuum Electronics Branch, Electronic Science and Technology Division, Naval Research Laboratory (NRL), Washington, DC. Since joining the Naval Research Laboratory in 1972, he has been actively involved in the stud of coherent radiation generation using beam-wave interactions in nonneutral plasma. In 1981, he was asked to form the Vacuum Electronics Branch, Electronics Science and Technology Division. Under his direction, an internal $R \& D$ program has been developed that addresses a wide range of issues affecting vacuum electronics. In addition to his role in internal R\&D efforts at the NRL, he is the Administrator for the Navy Applied Research program in Vacuum Electronics and the Chairman of the Tri-Service Vacuum Electronics Panel. He also serves as the Navy member on the Department of Defense (DoD) Advisory Group on Electron Devices (Working Group A). His personal research efforts have included an investigation of explosive field-emission diodes, the design and development of intense electron-beam accelerators, and the study of such beam-wave interaction mechanisms as the electron-cyclotron and free-electron masers, beam-front scattering, and the relativistic magnetron. He has authored or co-authored numerous scientific and technical publications in plasma physics, vacuum electronics, and accelerator technology.

Dr. Parker is a Fellow of the American Physical Society (Division of Plasma Physics). He currently serves as the chairman of the Executive Committee of the Nuclear and Plasma Science Society, Plasma Science and Applications Committee, and has served as an associate editor for the IEEE TRANSACTIONS ON ELECTRON DEVICES. He received the Navy Award for Group Achievement (1993) and the DoD Robert L. Woods Award (1994) for leadership in vacuum electronics. His other awards include the Navy Meritorious Civilian Service Award (1999) and the Capitol Club of the Association of Old Crows Technology Award (1998). 


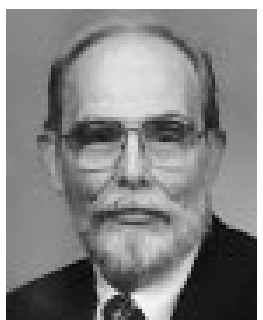

Richard H. Abrams, Jr. (A'93-M'93) received the B.S. degree in electrical engineering from the Massachusetts Institute of Technology (MIT), Cambridge, in 1959 , and the $\mathrm{Ph} . \mathrm{D}$. degree in plasma physics from the University of Maryland at College Park, in 1970

$\mathrm{He}$ is currently the Head of the Emission Physics Section, Vacuum Electronics Branch, Electronics Science and Technology Division, Naval Research Laboratory (NRL), Washington DC. He directs the Section's technical activities in advancing the state-of-the-art in innovative electron emitters for future vacuum electronic applications. His Section comprises the only DoD research capability for thermionic, secondary, and vacuum microelectronic electron emitters, which are vital to continued advances in performance of vacuum electronic devices. He was also instrumental in innovating the MPM Program and has served as the Program Manager of the Tri-Service MPM Program since its inception in 1991. He has been with the NRL since 1988 Prior to 1988 , he consulted for Government and industry, conducting technical assessments in vacuum electronics, high-power microwaves, GaAs MMICs and industrial energy use and conservation. He has supported Defense Science Board studies and was active in developing export control strategies for militarily critical electronics and computer technology.

Dr. Abrams was the recipient of the 1993 Navy Award for Group Achievement for leadership in vacuum electronics. His role in the innovation and development of the MPM was recognized by the 1995 Robert L. Woods Award, the 1996 NRL Technology Transfer Award, and his 2001 selection for the Association of Old Crows Technology Hall of Fame.

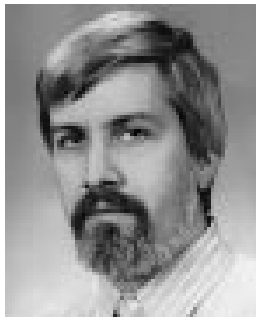

Bruce G. Danly (M'87-SM'01) received the B.A degree in physics from Haverford College, Haverford, PA, in 1978, and the Ph.D. degree in physics from the Massachusetts Institute of Technology (MIT), Cambridge, in 1983.

From 1983 to 1995 , he was on the research staff at the MIT Plasma Fusion Center, initially as a Research Scientist (1983-1992) and then as a Principal Scientist (1992-1995). While with MIT, he participated in research on gyrotrons, FELs, relativistic klystrons, and other high-power RF source technologies for use in plasma heating and high-gradient RF linear accelerators. In 1995, he joined the Naval Research Laboratory (NRL), Washington, DC, as Head of the High Power Devices Section, Vacuum Electronics Branch, Electronics Science and Technology Division. Technologies under investigation in this branch include gyrotron amplifiers (gyro-klystrons, gyrot-wystrons, gyro-TWTs), TWTs, an MPMs for communications, electronic warfare (EW), and radar applications.

Dr. Danly is a member of the IEEE Antennas and Propagation Society (IEEE AP-S). His role in the development of the high-power $W$-band gyro-klystron was recognized by the 1999 Robert L. Wood Award.

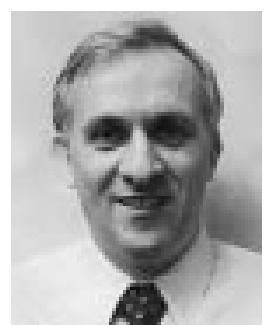

Baruch Levush (M'88-SM'90-F'01) received the M.Sc. degree in physics from the Latvian University, Riga, Latvia, and the Ph.D. degree in physics from Tel-Aviv University, Tel-Aviv, Israel.

In 1985, he joined the Institute for Plasma Research, University of Maryland at College Park, where his research focused on the physics of coherent radiation sources and the design of high-power microwave sources such as gyrotrons, TWTs, backward-wave oscillators, and FELs. In 1995, he joined the Naval Research Laboratory (NRL), Washington, DC, as the Head of the Theory and Design Section, Vacuum Electronics Branch, Electronics Science and Technology Division. $\mathrm{He}$ is actively involved in developing theoretical models and computational tools for analyzing the operation of vacuum electron devices and in inventing new concepts for high-power high-frequency coherent radiation sources. $\mathrm{He}$ is responsible for developing a suite of new design codes for vacuum electron devices under the auspices of the Office of Naval Research Modeling and Simulation Project. He has authored and co-authored over 130 journal papers.

Dr. Levush was the recipient of the 1999 DoD Robert L. Woods Award for his role in the successful development of a 10-kW average power $W$-band gyroklystron. 